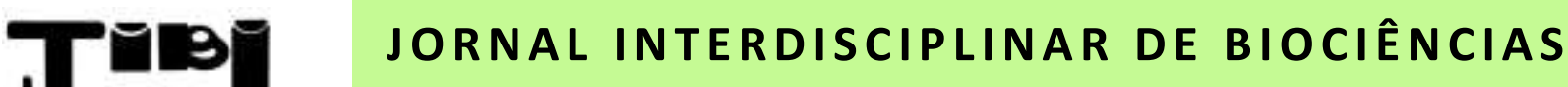

\author{
Homepage: http://www.ojs.ufpi.br/index.php/jibi
}

\section{Papel do sistema RANKL/RANK/OPG como regulador-chave da remodelação óssea durante a movimentação ortodôntica}

\section{RANKL/RANK/OPG system as a key regulator of bone remodeling during orthodontic tooth movement}

\author{
Rai Matheus Carvalho Santos ${ }^{1}$ e Cristiane Batista Bezerra Torres ${ }^{2 *}$ \\ ${ }^{1}$ Faculdade de Odontologia de Ribeirão Preto, Universidade de São Paulo; ${ }^{2 .}$ Departamento de Morfologia, Universidade Federal do \\ Piauí
}

\begin{abstract}
A B S T R A C T
RANKL, RANK and OPG have been considered as regulators of osteoclastogenesis. RANKL is produced by cells of bone or periodontal ligament in response to orthodontic forces. Its RANK membrane receptor is found in osteoclast precursor cells. Several cell types produce osteoprotegerin (OPG), which is a cytokine with affinity for RANKL and an inhibitory effect on it. The objective of the present work was to search for scientific evidences, through eletronic bibliographic research, of RANKL/RANK/OPG system roles in bone remodeling during orthodontic tooth movement. Papers were found at bibliographic databases such as LILACS, MEDLINE, PubMed and SciELO, using the search terms "rankl" and "orthodontic tooth movement". Research results suggest that RANKL activate osteoclastogenesis, with consequent acceleration of tooth movement, while OPG reduces movement by inhibiting the binding of RANKL to RANK. The RANKL and RANK control at the receptor or signaling pathway level may be an effective tool in the treatment of unwanted bone reabsorption, opening a promising field for new pharmacological approaches in orthodontic treatment and bone remodeling.
\end{abstract}

K E Y W O R D

RANKL, RANK, OPG, bone remodeling, orthodontic tooth movement

R E S U O

RANKL, RANK e OPG têm sido apontados como reguladores da osteoclastogênese. O RANKL é produzido por células ósseas ou do ligamento periodontal em resposta a forças ortodônticas. Seu receptor de membrana RANK é encontrado em células precursoras de osteoclastos. Vários tipos de células produzem osteoprotegerina (OPG), uma citocina com afinidade ao RANKL e efeito inibitório sobre ele. O objetivo do presente trabalho foi buscar evidências científicas, por meio de pesquisa bibliográfica, do papel do sistema RANKL/RANK/OPG na remodelação óssea durante a movimentação dentária ortodôntica. Artigos foram recuperados nas bases de dados bibliográficos LILACS, MEDLINE, PubMed e SciELO, por meio dos termos de busca "rankl" e "orthodontic tooth movement". Resultados de pesquisas sugerem que RANKL ativa a osteoclastogênese, com consequente aceleração do movimento do dente, enquanto a OPG o reduz, pela inibição da ligação RANKL/RANK. O controle de RANKL/RANK, em nível de receptores ou via de sinalização, pode ser uma ferramenta efetiva no tratamento de reabsorção óssea indesejada, abrindo campo promissor para novas abordagens farmacológicas no tratamento ortodôntico e remodelação óssea alveolar.

P A L A V R A S - C H A VE

RANKL, RANK, OPG, remodelação óssea, movimento dentário

\section{INTRODUÇÃO}

Modelos de movimentação ortodôntica são efetivos para o estudo da remodelação óssea induzida por forças mecânicas, as quais, quando aplicadas na coroa dentária, são transmitidas ao osso alveolar por meio do ligamento periodontal. É consenso, na literatura, que a aplicação dessas forças gera dois efeitos no osso: no sítio de compressão, a reabsorção óssea é induzida pela força da raiz sobre a parede óssea alveolar; no sítio de tensão, fibras do ligamento periodontal são esticadas, resultando em estímulo à formação de osso (CASTROFLORIO et al., 2017; NAKANO et al., 2011; SCHEPDAEL; SLOTEN; GERIS, 2013; TADDEI et al., 2012; ZHOU et al., 2011).

As alterações do tecido ósseo resultam da interação entre osteoblastos e osteoclastos. Células da linhagem 
osteoblástica não estão apenas envolvidas na formação óssea, mas também regulam a diferenciação de osteoclastos, bem como sua ativação e sobrevivência. Esses eventos são mediados pela liberação de várias moléculas, entre citocinas, neurotransmissores e fatores de crescimento, como resultado das alterações da microvascularização local. Desse modo, no sítio de compressão, haveria aumento da expressão de fatores da osteoclastogênese e, no lado da tensão, de fatores osteogênicos (SCHEPDAEL; SLOTEN; GERIS, 2013; TADDEI et al., 2012).

Os papéis de RANKL, RANK e OPG, na regulação da osteoclastogênese, têm sido extensivamente estudados desde sua descoberta na década de 1990. Desde então, foram evidenciadas suas importantes implicações na regulação de vários tecidos: remodelação óssea, formação de linfonodos, desenvolvimento de glândulas mamárias e controle da febre. Desordens no sistema RANKL/RANK/OPG estão associadas a certas doenças humanas, inclusive osteoporose pós-menopausa, artrite reumatoide, tumores ósseos e certos tumores metastáticos (BALOUL, 2016; LIU; ZHANG, 2015; THEILL; BOYLE; PENNINGER, 2002).

RANKL (ligante de RANK) é produzido como fator local por células do ligamento periodontal (osteoblastos e fibroblastos) em resposta a intensas forças ortodônticas. Recentemente, surgiram evidências de que os osteócitos são importante fonte de RANKL para a formação de osso esponjoso (XIONG et al., 2015). O receptor de membrana RANK (receptor activator of Nfk-B) é encontrado em células precursoras de osteoclastos. Vários tipos de células produzem osteoprotegerina (OPG), que é uma citocina secretada com afinidade ao RANKL e efeito inibitório sobre ele (NETTELHOFF et al., 2016; XING et al., 2017; ZHOU et al., 2011).

Alterações na concentração dessas moléculas, induzidas por variações dos níveis de outras substâncias, como hormônios (vitamina D3, paratormônio, estradiol), citocinas (IL-1, IL-6, IL-11, IL-17, TNF- $\alpha$ ), fatores de crescimento (TGF- $\beta$, BMP-2), prostaglandina E2 e glicocorticoides, poderiam influenciar o número e a atividade dos osteoclastos, com repercussões diretas sobre o tratamento ortodôntico (THEILL; BOYLE; PENNINGER, 2002). Desse modo, o estudo sobre a participação desse sistema na regulação da remodelação óssea alveolar poderia subsidiar novas abordagens farmacológicas para o tratamento de doenças que manifestam perda óssea e/ou reabsorção radicular.

O objetivo do presente trabalho é identificar evidências científicas do papel do sistema RANKL/RANK/OPG na remodelação óssea, durante a movimentação ortodôntica, por meio de pesquisa bibliográfica sobre o tema. Para tanto, uma busca eletrônica foi realizada nas bases de dados bibliográficos LILACS, MEDLINE, PubMed e SciELO, por meio dos termos de busca "rankl" e "orthodontic tooth movement”. Para a seleção de artigos úteis à discussão proposta, utilizou-se como critérios de inclusão a conexão direta ao tema e a disponibilidade do estudo na íntegra.

\section{DESENVOLVIMENTO}

A remodelação óssea alveolar é regida por fatores biológicos e mecânicos, sendo caracterizada por reações sequenciais do tecido periodontal em resposta às forças aplicadas sobre o elemento dentário por meio do tratamento ortodôntico (SCHEPDAEL; SLOTEN; GERIS, 2013). As modificações sofridas pelo tecido ósseo, durante esse processo, podem ser explicadas pela teoria da pressãotensão. No lado de pressão, onde ocorre a compressão dos tecidos, o osso alveolar sofre processo de reabsorção. Simultaneamente, no lado de tensão, onde há estiramento das fibras do ligamento periodontal, ocorre aumento do número de fibroblastos e osteoblastos e, consequentemente, síntese de colágeno e matriz óssea (SANTAMARIA et al., 2007; TADDEI et al., 2012; ZHOU et al., 2011).

Existem evidências de que essa regulação é mediada pelo RANKL, peptídeo com 317 aminoácidos e pertencente à família de fatores de necrose tumoral (TNF). O RANKL se liga ao RANK presente nas células progenitoras dos osteoclastos e, na presença de fator estimulante de colônia de macrófago (M-CSF), estimula a diferenciação e a atividade dos osteoclastos (NAKATO et al., 2011; ROGERS; EASTELL, 2005). Camundongos com deficiência em RANKL desenvolvem osteopetrose (LIU; ZHANG, 2015). A interação entre RANKL e seu receptor RANK, nos precursores de osteoclastos, seria controlada pela osteoprotegerina (OPG) - um receptor solúvel, com homologia aos receptores da família dos fatores de necrose tumoral (TNF) - que inibe a ligação do RANKL ao RANK, competindo com este pelo mesmo ligante, impedindo o recrutamento, a proliferação e a ativação dos osteoclastos (BEETON et al., 2006; LIU; ZHANG, 2015; TANG; LIN; LI, 2006; THÉOLEYRE et al., 2006).

Estudos demonstram que mudanças na tensão mecânica, provocada pelas forças ortodônticas, alteram os níveis de OPG e RANKL no ligamento periodontal (KANZAKI et al., 2006; NISHIJIMA et al., 2006; TADDEI et al., 2012) e tecido ósseo (XIONG et al., 2015). A expressão diferenciada desses mediadores, nos sítios de compressão e tensão, responderia por padrões específicos de migração celular e formação/reabsorção de tecido ósseo (LI et al., 2015; TADDEI et al., 2012; ZHANG et al., 2016; ZHOU et al., 2011). RANKL tem expressão aumentada, no lado da compressão, criando um ambiente propício para a 
reabsorção óssea. No lado da tensão, há aumento da expressão de marcadores de diferenciação osteoblástica (como o RUNX-2) e de reguladores negativos de osteoclastos (IL-10 e OPG), criando um microambiente favorável para a formação de tecido ósseo (BALOUL, 2016; TADDEI et al., 2012; ZHAO et al., 2012).

Essas variações de expressão molecular podem ser mensuradas também no fluido crevicular gengival (FCG). Estudos mostram que, após a aplicação da força ortodôntica, ocorrem isquemia e hipóxia no ligamento periodontal e no osso alveolar devido à compressão da microvascularização. Nesta fase inicial (24 horas a dois dias após a aplicação), a razão RANKL/OPG no FCG aumenta, favorecendo a osteoclastogênese, provavelmente pela redução dos níveis de OPG. Na fase de hialinização do ligamento, há redução da razão RANKL/OPG - pela maior expressão de OPG que de RANKL - e ausência de movimento dentário. Com o restabelecimento das funções celulares do ligamento, a expressão de OPG é inferior à de RANKL, com consequente reabsorção óssea e aceleração do movimento (NISHIJIMA et al., 2006; FLÓREZMORENO et al., 2013; CASTROFLORIO et al., 2017).

Como o RANKL parece ser o único ligante conhecido para o RANK, é essencial compreender o efeito da expressão desse peptídeo, sob condições normais e patológicas, no processo de remodelação óssea. Existem evidências de que paratormônio, prostaglandina E2, dexametasona, citocinas inflamatórias (IL-1, TNF- $\alpha$ ) e 1,25 diidrovitamina D3 estimulam a expressão de RANKL. Em contraste, estrogênio e TGF- $\beta$ atenuam sua expressão. Esses fatores supracitados geralmente têm efeitos opostos na expressão de RANKL e OPG (WADA et al., 2006).

A descoberta de que esses fatores (RANKL e RANK) são cruciais para a osteoclastogênese e ativação osteoclástica in vivo abriu as portas para o desenvolvimento de novas pesquisas na área de terapêutica medicamentosa (BALOUL, 2016; LIU; ZHANG, 2015). Embora IL-1 e TNF- $\alpha$ tenham sido propostos como efetivos no controle da perda óssea em artrites, são muito menos potentes que o RANKL em termos de indução de osteoclastos. Portanto, o controle de RANKL e RANK, em nível de receptores ou de via de sinalização, pode ser uma ferramenta vantajosa no tratamento de reabsorção óssea indesejada. Isso pode ser alcançado, por exemplo, com o uso de OPG recombinante e anticorpos anti-RANKL (KANZAKI et al., 2006; WADA et al., 2006, ZHAO et al., 2012). Este é um campo ainda aberto e promissor para novas descobertas farmacológicas no tratamento ortodôntico e de prevenção de reabsorção radicular.

\section{REFERÊNCIAS}

BALOUL, S. S. Osteoclastogenesis and osteogenesis during tooth movement. Frontiers of Oral Biology, v. 18, 75-9, 2016. BEETON, C. A.; BORD, S.; IRELAND, D.; COMPSTON, J. E. Osteoclast formation and bone resorption are inhibited by megakaryocytes. Bone, v. 39, n. 5, p. 985-990, 2006.

CASTROFlORIO, T.; GAMERRO, E. F.; CAVIGLIA, G. P.; DEREGIBUS, A. Biochemical markers of bone metabolism during early orthodontic tooth movement with aligners. The Angle Orthodontist, v. 87, n.1, 2017.

FLÓREZ-MORENO, G. A.; ISAZA-GUZMÁN, D. M.; TOBÓN-ARROYANE, S. I. Time-related changes in salivary levels of the osteotropic factors sRANKL and OPG through orthodontic tooth movement. American Journal of Orthodontics and Dentofacial Orthopedics, v. 143, p. 92-100, 2013.

KANZAKI, H.; CHIBA, M.; ARAI, K.; TAKAHASHI, I.; HARUYAMA, N.; NISHIMURA, M.; MITANI, H. Local RANKL gene transfer to the periodontal tissue accelerates orthodontic tooth movement. Gene Therapy, v. 13, p.678-685, 2006.

LI, B.; ZHANG, Y. H.; WANG, L. X.; LI, X.; ZHANG, X. D. Expression of OPG, RANKL, and RUNX2 in rabbit periodontium under orthodontic force. Genetics and Molecular Research, v. 14, n. 4, p. 19382-19388, 2015.

LIU, W.; ZHANG, X. Receptor activator of nuclear factor-KB ligand (RANKL)/RANK/osteoprotegerin system in bone and other tissues (review). Molecular Medicine Reports, v. 11, p. 3212-3218, 2015.

NAKANO, Y.; YAMAGUCHI, M.; FUJITA, S.; ASANO, M.; SAITO, K.; KASAI, K. Expressions of RANKL/RANK and M$\mathrm{CSF} / c$-fms in root resorption lacunae in rat molar by heavy orthodontic force. European Journal of Orthodontics, v. 33, p. 335-343, 2011.

NETTELHOFF, L.; GRIMM, S.; JACOBS, C.; WALTER, C.; PABST, A. M.; GOLDSCHIMITT, J.; WEHRBEIN, H. Influence of mechanical compression on human periodontal ligament fibroblasts and osteoblasts. Clinical Oral Investigations, v. 20, n. 3, p. 621-629, 2016

NISHIJIMA, Y.; YAMAGUCHI, M.; KOJIMA, T.; AIHARA, N.; NAKAJIMA, R.; KASAI, K. Levels of RANKL/OPG in gingival crevicular fluid during orthodontic tooth movement and effect of compression force on releases from periodontal ligament cells in vitro. Orthodontics and Craniofacial Research, v. 9, p. 63-70, 2006.

SANTAMARIA JR., M; MILAGRES, D; LYOMOSA, M. M.; STUANI, M. B.; RUELLAS, A.C. Initial pulp changes during orthodontic movement: histomorphological evaluation. Brazilian Dental Journal, v. 18, n. 1, p. 34-39, 2007.

SCHEPDAEL, A. V.; SLOTEN, J. V.; GERIS, L. A mechanobiological model of orthodontic tooth movement. Biomechanics and Modeling in Mechanobiology, v. 12, p. 249$265,2013$.

TADDEI, S. R. A.; MOURA, A. P.; ANDRADE JR., I.; GARLET, G. P.; GARLET, T. P.; TEIXEIRA, M. M.; SILVA, T. 
A. Experimental model of tooth movement in mice: a standardized protocol for studying bone remodeling under compression and tensile strains. Journal of Biomechanics, v. 45, p. 2729-2735, 2012.

TANG, T.; LIN, Z.; LI, Y. M. Effects of different magnitudes of mechanical strain on osteoblasts in vitro. Biochemical and Biophysical Research Communications, v. 344, n. 1, p. 122$128,2006$.

THEILL, L. E.; BOYLE, W. J.; PENNINGER, J. M. RANK-L and RANK: T cells, bone loss, and mammalian evolution. Annual Review of Immunology, v. 20, p. 795-823, 2002.

THÉOLEYRE, S., KWANTAT, S., VUSIO, P., BLANCHARD, F., GALLAGHER, J., RICARD-BLUM, S.; FORTUN, Y.; PADRINES, M.; REDINI, F.; HEYMANN, D. Characterization of OPG binding to glycosaminoglycans by surface plasmon resonance: role in the interactions with RANKL/RANK. Biochemical and Biophysical Research Communications, v. 347, n. 2, p. 460-467, 2006.

WADA, T.; NAKASHIMA, T.; HIROSHI, N.; PENNINGER, J.M. RANKL-RANK signaling in osteoclastogenesis and bone disease. TRENDS in Molecular Medicine, v. 12, n. 1, p. 17-25 2006.

XING, J. Z.; LU, L.; UNSWORTH, L. D.; MAJOR, P. W.; DOSCHAK, M. R.; KAIPATUR, N. R. RANKL release from self-assembling nanofiber hydrogels for inducing osteoclastogenesis in vitro. Acta Biomaterialia, v. 49, p. 306315, 2017.

XIONG, J.; PIEMONTESE, M.; ONAL, M.; CAMPBELL, J.; GOELLNER, J. J.; DUSEVICH, V.; BONEWALD, L.; MANOLAGAS, S. C.; O'BRIEN, C. A. Osteocytes, not osteoblasts or lining cells, are the mains source of the RANKL required for osteoclast formation in remodeling bone. PLoS ONE, v. 10, n. 9, 2015.

ZHANG, L.; LIU, W.; ZHAO, J.; MA, X.; SHEN, L.; ZHANG, Y.; JIN, F.; JIN, Y. Mechanical stress regulates osteogenic differentiation and RANKL/OPG ration in periodontal ligament stem cells by the Wnt/ $\beta$-catenin pathway. Biochimica et Biophysica Acta. v. 1860, n. 10, p. 2211-2219, 2016.

ZHAO, N; LIN, J.; KANZAKI, H.; NI, J.; CHEN, Z.; LIANG, W.; LIU, Y. Local OPG gene transfer inhibits relapse of orthodontic tooth movement. American Journal of Orthodontics and Dentofacial Orthopedics, v. 141, p. 30-40 2012.

ZHOU, J; FENG, G.; ZHOU, W.; REN, A.; WU, Y; ZHANG, D.; DAI, H. Expression of osteoprotegerin and receptor activator of nuclear factor $\mathrm{\kappa B}$ ligand in root resorption induced by heavy force in rats. Journal of Oral Orthopedics, v. 72 , n. 6, p. 457-468, 2011. 